\title{
Regulação da massa corpórea pelo estrogênio e pela atividade física
}

\author{
Body mass regulation by estrogen and physical activity
}

Daniele L. Ignacio', Tamar G. P. Frankenfeld', Rodrigo S. Fortunato', Mário Vaisman², João Pedro Saar Werneck-de-Castro ${ }^{3}$, Denise P. Carvalho'

'Laboratório de Fisiologia Endócrina, Instituto de Biofísica Carlos Chagas Filho (IBCCF), Universidade Federa do Rio de Janeiro (UFRJ), Rio de Janeiro, RJ, Brasil ${ }^{2}$ Serviço de Endocrinologia, Hospital Universitário Clementino Fraga Filho (HUCFF), UFRJ, Rio de Janeiro, RJ, Brasil ${ }^{3}$ Laboratório de Biologia Muscular e do Exercício, Departamento de Biociências da Atividade Física, UFRJ, Rio de Janeiro, RJ, Brasi

Correspondência para:

Denise P. Carvalho IBCCF da UFRJ

CCS - Bloco G - Cidade Universitária 21941-902 - Rio de Janeiro, RJ, Brasil

dencarv@biof.ufri.br

Recebido em 2/Fev/2009

Aceito em 2/Abr/2009

\begin{abstract}
RESUMO
A deficiência de esteroides gonadais femininos acelera o ganho de massa corpórea, mas os possíveis mecanismos centrais e periféricos envolvidos no aumento da ingestão alimentar e no ganho de massa adiposa que ocorrem nessa condição são pouco conhecidos. Em modelos animais, tanto a falta quanto os defeitos na ação do estrogênio causam aumento da massa corpórea, demonstrando claramente um possível papel desse esteroide no sobrepeso pós-menopausa. Sabe-se que a obesidade e o sobrepeso estão associados a diversas comorbidades que podem levar à morte prematura. Portanto, desvendar os mecanismos relacionados ao ganho de massa corpórea é de grande relevância, assim como desenvolver estratégias que possam prevenir o seu estabelecimento. A regulação do balanço energético está associada ao controle da massa corpórea, sendo o exercício físico um importante modulador desse parâmetro homeostático. Porém, a influência do exercício físico sobre o ganho de massa corpórea durante a deficiência de estrogênio é controversa e depende do protocolo de exercício utilizado. Neste estudo, pretendemos revisar os achados que relacionam a deficiência de estrogênio ao ganho de massa corpórea em animais e seres humanos. Arq Bras Endocrinol Metab. 2009;53(3):310-7.

Descritores

Sobrepeso; exercício; estrogênio; menopausa; ovariectomia
\end{abstract}

\begin{abstract}
Female steroid hormones deficiency leads to a significant increase in body mass, but the possible central and peripheral mechanisms involved in increased food ingestion and fat accumulation in this situation are still unknown. In animal models, the specific lack of estrogen or its action produce progressive body mass gain, clearly demonstrating the possible role of this hormone in overweight after menopause. Obesity and overweight correspond to a relevant human health problem that can lead to premature death. Therefore unraveling the mechanisms underlying body mass gain is of great relevance, as well as the development of strategies to prevent its establishment. Energy balance regulation is associated with the control of body mass, and physical exercise is an important modulator of this homeostatic parameter. However, the influence of physical exercise in mass gain development during estrogen deficiency is controversial and depends on the exercise protocol used. In this study, we intend to review the data on the effects of estrogen deficiency on body mass gain in humans and animal models. Arq Bras Endocrinol Metab. 2009;53(3):310-7.

Keywords

Overweight; exercise; estrogens; menopause; ovariectomy
\end{abstract}

\section{ESTEROIDES SEXUAIS FEMININOS}

W strogênio é um termo usado para um grupo de Chormônios esteroides com 18 carbonos, que são secretados principalmente pelo ovário e, em menor quantidade, pelas adrenais. $\mathrm{O}$ estrogênio engloba três hormônios esteroides estruturalmente semelhantes: 17ß-estradiol (E2), estrona (E1) e estriol (E3). Desses, o $17 \beta$-estradiol é o principal esteroide em humanos que possui propriedades estrogênicas (1).

A secreção dos hormônios gonadais é regulada pelo eixo hipotálamo-adeno-hipófise. Em resposta ao hormônio liberador de gonadotrofinas $(\mathrm{GnRH})$, a hipófi- 
se anterior secreta os hormônios folículo estimulante (FSH) e luteinizante (LH). No sexo feminino, a função do FSH é regular o crescimento folicular e a produção crescente de estradiol pelas células da granulosa. $\mathrm{O}$ LH é responsável por aumentar a captação de colesterol e estimular as células intersticiais da teca dos folículos ovarianos a secretar androstenediona e testosterona (androgênios). Após a ovulação, FSH e, principalmente, LH agem nas células luteinizadas da granulosa e da teca do folículo rompido (corpo lúteo), aumentando a produção de estradiol e, em maior quantidade, a de progesterona. Nesta fase, os hormônios gonadais regulam a secreção de GnRH, FSH e LH por meio de um mecanismo de retroalimentação negativa. Peptídeos produzidos no ovário, como a inibina e a ativina, também modulam negativamente a secreção de FSH (2).

\section{DEFICIÊNCIA DE ESTROGÊNIO E CONTROLE DA MASSA CORPÓREA}

Desde a década de 1980, sabe-se que, em ratas, a ovariectomia acelera o ganho de massa corpórea, mais especificamente de tecido adiposo ou de proteínas (3). Esses animais, além do aumento de massa corpórea, apresentaram também maior ingestão alimentar - alterações que foram impedidas pela reposição com E2. A menor massa corpórea dos animais repostos com E2 denota um possível efeito lipolítico do estrogênio e a inibição do neuropeptídeo $\mathrm{Y}$, com consequente diminuição da ingestão alimentar $(4,5)$. Recentemente, confirmamos esses achados prévios e iniciamos estudos relacionados ao período durante o qual o sobrepeso ainda não se instalou, com o intuito de definirmos quais seriam os possíveis fatores humorais relacionados ao aumento da massa corpórea nas ratas ovariectomizadas $(6,7)$.

No entanto, o papel inequívoco do estrogênio na regulação da gordura corporal foi confirmado em estudos com camundongos deficientes do receptor $\alpha$ de estrogênio (ER- $\alpha$ ) ou da aromatase (Aro), enzima responsável pela biossíntese de estrogênio, que apresentam aumento do tecido adiposo branco $(8,9)$. Entretanto, os mecanismos pelos quais o E2 exerce influência sobre o comportamento alimentar e a regulação da massa corpórea não são claros. A deficiência de estrogênio pode estar relacionada à diminuição de receptores de leptina no hipotálamo, o que causaria diminuição da saciedade (4), maior ingestão e consequente ganho de massa corpórea. Por outro lado, há também possibilidade de diminuição do gasto energético em fềmeas com deficiência de estrogênio, fato que facilitaria o ganho de massa corpórea (10).

\section{DEFICIÊNCIA DE ARO}

A etapa final da biossíntese de estrogênio é catalisada por uma enzima microssomal que faz parte da superfamília do citocromo P450, chamada citocromo aromatase P450 (P450arom, produto do gene Cyp19) (11).

Camundongos fêmeas com deleção do gene da Aro possuem a produção de estrogênio bloqueada e apresentam alterações endócrinas severas no sistema reprodutor, acúmulo de tecido adiposo e alterações em nível esquelético, tais como perda do volume e espessura do osso trabecular e osteopenia (12).

Embora pareça que o estrogênio tenha um papel crucial no crescimento do ovário, outros parâmetros são também alterados nesse modelo, como o aumento das concentrações séricas de testosterona, LH e FSH (13). Nesses animais, há aumento de gordura intraabdominal e diminuição de massa magra, além de alterações no perfil lipídico (9).

A deficiência de Aro em humanos é uma doença rara, com apenas poucos casos descritos em homens e mulheres (14). O estudo dos efeitos do tratamento com estrogênio nesses casos e a investigação de camundongos com deleção no gene do receptor de estrogênio e da Aro têm contribuído para o entendimento do papel do estrogênio na fisiologia do controle do sobrepeso (15). Até agora, as ações mais conhecidas do estrogênio em homens são as relacionadas à manutenção e à aquisição da massa óssea (16), ao fechamento das epífises (17), à adiposidade e à síndrome metabólica (18), além da modulação da secreção das gonadotrofinas (19). Outras características singulares associadas à deficiência congênita de estrogênio sugerem um papel mais complexo deste esteroide em homens. Essas características clínicas incluem uma complexa síndrome metabólica com vários graus de sobrepeso, hiperinsulinemia, de moderada à severa, e metabolismo de glicose e lipídeo prejudicados $(17,20)$. Esses achados estão de acordo com os encontrados em camundongos com deleção do gene da Aro (14).

No trabalho de Lanfranco e cols. (21), foi descrito um caso de deficiência de Aro em um homem com 26 anos de idade. O paciente foi submetido a um tratamento de reposição hormonal com estradiol transdérmico, que não foi capaz de diminuir a massa corpórea, tampouco a adiposidade (2l), o que também foi ob- 
servado no estudo de Maffei e cols. (17). Isso sugere que o tratamento com estrogênio pode não ser eficiente para reverter a obesidade nesses pacientes (21). Entretanto, Maffei e cols. (17) descreveram o aumento de densidade mineral óssea e a normalização nos níveis de marcadores de reabsorção óssea durante tratamento com estradiol. Além disso, a reposição com estrogênio teve um efeito positivo no metabolismo da glicose, melhorando a sensibilidade à insulina, apesar do aumento da massa corpórea, e reforçando os efeitos positivos do estrogênio no metabolismo da glicose (17) e, mais uma vez, confirmando os dados encontrados em camundongos $\operatorname{ArKO}(14)$.

Atualmente, a importância do estrogênio para a maturação óssea em homens deficientes em Aro é conhecida, porém a quantidade de estradiol necessária para o completo fechamento das epífises não o é (21). No estudo de Maffei e cols. (17), níveis séricos de estradiol acima de $73 \mathrm{pmol} / \mathrm{L}(20 \mathrm{pg} / \mathrm{mL})$ durante o tratamento de reposição com estrogênio em homens com deficiência de Aro resultou num rápido crescimento das extremidades e em fechamento das epífises ósseas, enquanto, no paciente do estudo de Lanfranco e cols. (21), apresentou níveis séricos de estradiol de 60,6 pmol/L (16,5 pg/mL), a completa maturação óssea não foi alcançada $(21)$.

Mulheres no período da menopausa também possuem desbalanço no metabolismo ósseo, com predomínio da reabsorção óssea sobre a formação, o que leva à perda de massa óssea (22). É digno de nota que o declínio na produção de estrogênio é um dos principais fatores determinantes desse desbalanço $(23,24)$.

\section{ANIMAIS COM DELEÇÃO NOS GENES DOS RECEPTORES DE ESTROGÊNIO}

As respostas fisiológicas ao estrogênio são mediadas por, pelo menos, dois tipos de receptores de estrogênio (ERs): ER $\alpha$ e ER $\beta$. Os ERs são membros de uma ampla família de receptores hormonais nucleares estimulados pelos ligantes (24). Porém, há crescentes evidências de que o estrogênio, assim como outros esteroides, possa modular funções celulares por meio de ações não genômicas (25).

$\mathrm{O}$ padrão tecidual de expressão dos receptores indica uma ampla distribuição do $E R \alpha$, enquanto o $E R \beta$ possui distribuição mais limitada, com alta expressão no ovário, próstata, epidídimo, pulmão e hipotálamo. As respostas fisiológicas exatas atribuídas a cada receptor, na sua maioria, são desconhecidas (26).
A falta de ativação do ER implica metabolismo de glicose e lipídeos prejudicado na pós-menopausa, o que resulta em aumento da adiposidade visceral, resistência insulínica e aumento do risco para doenças cardíacas (27).

Camundongos com deleção nos genes dos ERs são modelos interessantes para o estudo dos mecanismos reguladores do metabolismo e da composição corporal. Camundongos com deleções do ER $\alpha$ têm aumento na massa de tecido adiposo branco, bem como resistência à insulina e diminuição da tolerância à glicose, em ambos os sexos $(8,28)$.

A função metabólica do $\operatorname{ER} \beta$ não é clara. $\mathrm{O}$ animal deficiente em ER $\beta$ (ER $\beta K O)$ possui massa corpórea e distribuição de gordura normais (29). Um estudo recente de Foryst-Ludwig e cols. (30) demonstrou pela primeira vez que o ER $\beta$ diminui a sensibilidade à insulina $\mathrm{e}$ a tolerância à glicose de animais submetidos a uma dieta rica em gordura, indicando ações pró-diabetogênicas desse receptor. Recentemente, o mesmo grupo mostrou que o ER $\beta$ possui um papel supressor na expressão do transportador 4 de glicose (GLUT 4) no músculo esquelético (31). O GLUT4 é considerado o maior mediador da captação de glicose induzida por insulina no músculo esquelético e o tecido adiposo (32). Entretanto, a ausência do ER $\beta$ impediu o aumento de triglicerídeos e manteve a sinalização de insulina preservada em fígado e músculo esquelético. Assim, esse estudo mostra que o ER $\beta$ prejudica o metabolismo da glicose e insulina, o que pode ser resultado, pelo menos em parte, de um crosstalk negativo com o receptor de ativação de proliferação de peroxissomo (PPAR $\gamma$ ) presente nos adipócitos (30).

$\mathrm{O}$ receptor nuclear órfão denominado ERR $\alpha$ tem relação com a sinalização dos estrogênios na formação óssea e no metabolismo oxidativo. Em níveis fisiológicos, o ERR $\alpha$ é essencial para a sobrevivência em situações de aumento da demanda energética. A atividade diminuída do ERR $\alpha$ reduz a capacidade de gerar energia, ocorrendo redução da capacidade física e da sobrevivência em situações de estresse metabólico (33).

\section{MENOPAUSA E DISTRIBUIÇÃO DE GORDURA CORPORAL}

A diminuição nos níveis de estrogênio em mulheres na pós-menopausa associa-se com o desenvolvimento de doenças crônicas, tais como osteoporose e doenças cardiovasculares. Comparadas com os homens, as mulheres têm menor acúmulo central de gordura corporal (34). Entretanto, a maioria dos estudos demonstrou 
aumento na gordura abdominal em mulheres durante a transição para a menopausa (27).

O tecido adiposo é um órgão endócrino ativo que, além de regular a massa gorda e a homeostase dos nutrientes, libera uma série de mediadores bioativos (adipocitocinas), que regulam a homeostase, a pressão sanguínea, o metabolismo da glicose e dos lipídeos, inflamação e aterosclerose (34).

No estudo de Hong e cols. (35), foi investigada a associação entre adipocitocinas séricas (adiponectina, resistina, leptina e TNF $\alpha$ ) e os níveis séricos de estrogênio (estrona e etradiol) em mulheres saudáveis na pré e pósmenopausa. Os níveis de TNF $\alpha$ foram significativamente maiores após a menopausa, sugerindo que a deficiência de estrogênio resulta no aumento de citocinas inflamatórias séricas, o que pode contribuir para o desenvolvimento de aterosclerose e diabetes melito tipo 2 (35).

A leptina sérica normalizada pelo índice de massa corpórea (IMC) foi menor na pós-menopausa, indicando que os níveis de leptina se correlacionam positivamente com os níveis de estrogênio. Entretanto, os níveis absolutos de leptina sérica estavam aumentados como a massa corpórea, o que pareceu contribuir significativamente para a resistência insulínica. Os níveis de adiponectina se correlacionam negativamente com o IMC e com a resistência insulínica, mas os mecanismos que determinam esses achados não estão claros (35). Uma hipótese é a de que o TNF $\alpha$, presente no tecido adiposo branco de indivíduos obesos, possa inibir a produção de adiponectina (36). O papel fisiológico da resistina na obesidade e na resistência insulínica não é claro. Em animais, estudos mostram um efeito inibitório da resistina na captação de glicose estimulada por insulina (37) e, em humanos, o aumento da resistina associa-se à obesidade e à resistência insulínica (37). Foi visto também em animais, in vivo e in vitro, que o estrogênio diminui os níveis de RNAm para resistina em adipócitos (38).

No estudo de Hong e cols. (35), a adiponectina não teve correlação com TNF $\alpha$ sérico, assim como não teve correlação com os níveis de estrogênio após ajuste do IMC. Portanto, os mecanismos envolvidos na regulação da adiponectina pelo estrogênio não são claros (35).

Finalmente, a resistina sérica não se alterou significativamente quando comparada entre as mulheres, antes e depois da menopausa, normalizando pelo IMC ou não. Esse fato sugere que os níveis circulantes de resistina não possuem papel fundamental na resistência insulínica e não é regulada pelo estrogênio em humanos (35).
Diversos tipos de tratamento com reposição hormonal mostraram ser eficientes na prevenção do aumento de adiposidade central em mulheres durante o período da pós-menopausa quando comparadas a mulheres que não recebiam tratamento (39). Os estudos no período de transição da menopausa e terapias de reposição hormonal mostram uma mudança significativa no padrão de distribuição de gordura. Mais especificamente, a deficiência de estrogênio parece acelerar o acúmulo de gordura abdominal (40). O trabalho de Rebuffé-Scrive e cols. (40) descreve menor atividade da lipase lipoproteica (LPL) em mulheres na menopausa em tecido adiposo femoral, mas não da LPL abdominal. $\mathrm{O}$ tratamento hormonal com estrogênio isolado ou estrogênio combinado com progesterona estimula a atividade da LPL na região femoral, o que demonstra a influência regional específica do estrogênio sobre o tecido adiposo $(40)$.

\section{EXERCÍCIO FíSICO E CONTROLE DA MASSA CORPÓREA}

O estudo do controle de massa corpórea está essencialmente ligado à regulação do balanço energético, que depende de dois componentes básicos: a energia consumida e a energia gasta. Quando a energia consumida é equivalente a que se gasta, a massa corpórea permanece constante (41). Entretanto, o ganho de massa corpórea, em adultos saudáveis, ocorre quando a energia consumida excede persistentemente o gasto energético (42). A alta prevalência de obesidade e a sua associação com diabetes melito, hipertensão, hiperlipidemia e doenças cardiovasculares significam graves problemas de Saúde Pública (41). A prevenção e/ou o tratamento da obesidade requer a redução do consumo alimentar e/ou o aumento do gasto energético (43).

O gasto energético diário total é composto pela taxa metabólica basal (TMB), termogênese pós-prandial e termogênese induzida pela atividade física. Esta última se subdivide na termogênese que acompanha o exercício físico e na termogênese que acompanha as atividades da vida diária ou atividade física não relacionada ao exercício (do inglês non exercise activity thermogenesis, NEAT). TMB é o mínimo de energia que um indivíduo necessita para manter as funções vitais do organismo em estado de repouso pós-absortivo, em situação de termoneutralidade. Termogênese pós-prandial é o aumento do gasto energético relacionado à digestão, à absorção e ao estoque de alimentos (44). A atividade 
física é o componente do gasto energético total mais variável e o mais fácil de ser alterado (45). A regulação da atividade física diária não é completamente entendida, principalmente por ser afetada por uma série de componentes regulatórios (43) (Figura 1).

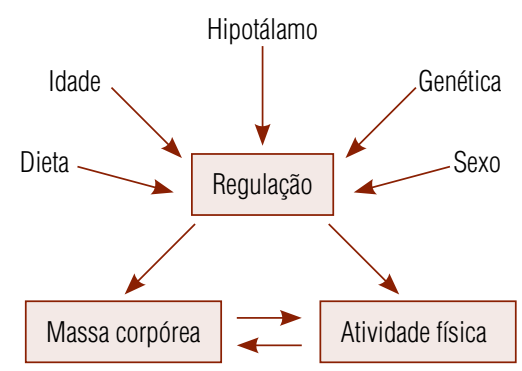

Figura 1. Componentes regulatórios que afetam o nível de atividade física não relacionada ao exercício. Adaptado de Tou e Wade (43).

Entende-se por atividade física qualquer movimento voluntário, produzido pelos músculos esqueléticos, que resulta em aumento do gasto energético (42). Exercício físico, por sua vez, é definido como qualquer atividade física planejada, estruturada e repetitiva, com o intuito de melhorar ou manter um ou mais componentes da aptidão física ou do rendimento (45).

Um estudo com ratos Zucker obesos (340 a $400 \mathrm{~g}$ ) mostrou que esses animais, quando submetidos ao exercício físico em esteira rolante, suportavam correr numa velocidade que era apenas a metade da velocidade que alcançavam seus pares selvagens não obesos ( 175 a $200 \mathrm{~g}$ ), indicando que a capacidade física diminuída pode ser secundária ao aumento de massa corpórea, porém, isso ainda é controverso (46).

\section{DEFICIÊNCIA DE ESTROGÊNIO E CONTROLE DA MASSA CORPÓREA: 0 PAPEL DA ATIVIDADE FÍSICA}

Mulheres na pós-menopausa possuem tendência a ganhar massa corpórea. Durante essa fase da vida, há diminuição nos níveis de atividade física, levando à redução do gasto energético, o que acarreta aumento de adiposidade abdominal e pode agravar as complicações metabólicas da obesidade (47). Porém, não se sabe exatamente como a menopausa contribui para a diminuição do nível de atividade física. Apenas metade das mulheres com idade entre 50 e 64 anos fazem alguma atividade física regular, e apenas um quarto relatam a prática de exercícios de alta intensidade. Assim, a diminuição da atividade física pode ser um importante fator para o ganho de massa corpórea após a menopausa (48).

Os componentes do gasto energético possuem um importante papel no aumento da adiposidade central e de todo o corpo que acontece em mulheres após a menopausa. Como demonstrado em estudo de Poehlman, Toth e Gardner (47), mulheres que entram na menopausa apresentam maiores reduções da TMB (aproximadamente $100 \mathrm{kcal} /$ dia) comparadas às que não estão na menopausa. Os níveis de atividade física também são menores em mulheres na menopausa (aproximadamente $127 \mathrm{kcal} / \mathrm{dia})$. As reduções da TMB e dos níveis de atividade física podem estar relacionadas, pelo menos em parte, à diminuição da massa magra. O gasto energético reduzido associado a nenhuma mudança ou ao aumento da ingestão alimentar gera um balanço energético positivo, fato que favorece o aumento da gordura corpórea total e, possivelmente, da adiposidade central (47).

Estudos prévios sugerem que o climatério seja um período durante o qual o gasto energético e a utilização dos substratos energéticos estão desregulados, com menor oxidação de gordura. Isso pode ser devido a alterações nos níveis de estrogênio e/ou androgênio, na massa livre de gordura reduzida e mudanças na ingestão alimentar induzidas pela deficiência de estrogênio (49).

No estudo de Kohrt, Ehsani e Birge (50), foram investigadas mudanças na composição corporal e na distribuição de gordura corporal em resposta ao exercício físico, à terapia de reposição hormonal (estrógenos conjugados e medroxiprogesterona) isolados, e à terapia aliada ao exercício físico. O estudo consistiu em um período de tratamento de 11 meses com avaliações antes do tratamento, ao final do tratamento e seis meses após seu término. As mulheres que participaram somente do programa de exercício e as que fizeram tratamento hormonal em conjunto com o exercício físico tiveram reduções na circunferência do quadril após 12 meses, porém, as mulheres que aliaram tratamento hormonal ao exercício físico tiveram menores circunferências de quadril após seis meses do término do tratamento comparadas às mulheres que somente se exercitaram. Isso sugere que a reposição hormonal pode ser usada em conjunto com o exercício físico para prevenir mudanças na distribuição de gordura que acompanham a menopausa. (50).

Com relação aos efeitos do estrogênio sobre o gasto energético, em ratos, acredita-se que esse esteroide seja capaz de regular a expressão de proteínas desacopladoras da mitocôndria (UCPs) nos tecidos adiposo branco e 
marrom. Ratas ovariectomizadas apresentam redução na expressão de UCPl no tecido adiposo marrom, entretanto, a expressão de UCP2 e UCP3 não se altera (51).

Observa-se também redução na expressão de UCP2 no tecido adiposo branco de ratas ovariectomizadas. A redução na expressão das UCPs 1 e 2 nesses diferentes tipos de tecidos poderia ser um dos fatores responsáveis pelo decréscimo no gasto energético e consequente aumento da massa corpórea em ratas ovariectomizadas (51).

Há dimorfismo sexual em relação ao nível de atividade física em roedores (42), pois as fềmeas são mais ativas que os machos (52). Uma das hipóteses para o dimorfismo sexual são os altos níveis de estrogênio observados nas fềmeas, já que o papel do estrogênio na regulação da atividade física pode ser observado por meio de mudanças hormonais que acompanham o ciclo estral de ratas. Durante a fase do proestro, em que os níveis plasmáticos de estrogênio estão elevados, há aumento na atividade física. Por outro lado, na fase do diestro, quando os níveis de estrogênio estão baixos, ocorre diminuição na atividade física dos animais (53).

Estudos com ratas ovariectomizadas também reforçam a importância do estrogênio na regulação da atividade física. Ratas ovariectomizadas tiveram diminuição da atividade voluntária de corrida. Porém, quando tratados com doses fisiológicas de estrogênio, esses animais recuperavam o nível de atividade física que tinham antes da ovariectomia (53).

Por outro lado, o ganho de massa corpórea parece regular a atividade física. Shimomura e cols. (54) mostraram que não houve mudanças na ingestão alimentar de ratas ovariectomizadas que apresentaram aumento de massa corpórea e diminuição da atividade, quando comparadas às do grupo controle. Assim, o ganho de massa corpórea poderia ser causado pela redução do gasto energético e pelo decréscimo gradual na atividade locomotora de ratas ovariectomizadas. Há explicações que justificam a regulação da atividade física pela massa corpórea. Estudos com roedores mostram que existem mediadores centrais e humorais que regulam a atividade física diária não relacionada a exercícios físicos; um destes mediadores é a orexina. Esse neuropeptídeo, quando foi injetado no núcleo paraventricular do hipotálamo, aumentou a atividade física espontânea em ratos (55).

Muitos hormônios e neuropeptídeos que afetam o apetite também modulam a atividade física, incluindo neuromedina $U$ e orexina, e a obesidade pode representar uma condição associada ao decréscimo da sensibilidade central a esses neuropeptídeos, os quais são responsáveis por aumentar a atividade física e, consequentemente, o gasto energético (56).

No estudo de Richard, Rochon e Deshaies (3), para verificar as possíveis interações entre exercício físico e hormônios ovarianos no balanço energético de ratas ovariectomizadas, foi observado que a ovariectomia aumenta a ingestão alimentar e a massa corpórea por aumento do conteúdo de proteína na carcaça, efeitos que foram revertidos pelo tratamento com benzoato de estradiol. O exercício crônico em esteira rolante por 33 dias não alterou esses parâmetros. Para esses autores, é provável que o ganho de massa corporal se deva ao aumento da ingestão, já que a termogênese no tecido adiposo marrom nas ratas tratadas ou não com estrogênio não se alterou após esse tipo de exercício físico.

Latour, Shinoda e Lavoie (57) mostraram que o exercício regular em esteira rolante durante oito semanas não reduziu o ganho de massa corpórea ou a ingestão alimentar de ratas ovariectomizadas e intactas tratadas ou não com estrogênio, porém, isso não significa que a composição corporal não tenha sido alterada com o treinamento. Já no estudo de Melton e cols. (58), o treinamento de natação em ratas ovariectomizadas, durante oito semanas, impediu o ganho de massa corpórea após ovariectomia, apesar do aumento da ingestão alimentar.

Embora a terapia de reposição hormonal seja amplamente utilizada para reduzir o risco de doença cardiovascular durante a menopausa, seu uso como um agente para regular o fenótipo na pós-obesidade começou a ser investigado apenas recentemente. Evidências preliminares sugerem que o exercício físico e/ou intervenções farmacológicas para compensar os prejuízos metabólicos e os riscos de doença cardíaca no período de transição para a menopausa ou após a menopausa são interessantes (59).

\section{CONSIDERAÇÕES FINAIS}

A regulação do balanço energético é fundamental para o controle da massa corpórea e a obesidade reflete o desbalanço energético crônico. $\mathrm{O}$ papel do estrogênio na regulação da gordura corporal foi confirmado por meio de estudos em animais $(8,28)$ e em seres humanos $(17,21)$. Mulheres na menopausa tendem a ganhar mais peso e modificar o padrão de distribuição de gordura corporal. Sabe-se que adipocinas liberadas pelo tecido adiposo podem contribuir para o desenvolvimento de comorbidades, como aterosclerose e diabetes melito tipo 2 (35). Durante essa fase da vida, há diminuição 
nos níveis de atividade física, contribuindo para a redução do gasto energético e para o aumento de adiposidade abdominal que pode agravar as complicações metabólicas da obesidade (48). Porém, não se sabe exatamente como a menopausa contribui para a diminuição do nível de atividade física. Em ratos, acredita-se que o estrogênio seja capaz de regular a expressão de proteínas desacopladoras da mitocôndria (UCPs) nos tecidos adiposo branco e marrom (51). Porém, a massa corpórea também pode regular a atividade física, visto que a quantidade de tecido adiposo é inversamente proporcional ao nível de atividade física (60). Neuropeptídeos, como a orexina e neuromedina $\mathrm{U}$, modulam a atividade física, e a diminuição da sensibilidade central a estes neuropeptídeos pode estar associada à obesidade (58). No entanto, a relação entre atividade física e deficiência de estrogênio ainda merece mais estudos que avaliem o papel integrado do estrogênio e do treinamento físico sobre a regulação da massa corpórea.

Financiamento: Programa de Apoio a Núcleos de Excelência (PRONEX)/Fundação de Amparo à Pesquisa do Estado do Rio de Janeiro (FAPERJ), Conselho Nacional de Desenvolvimento Científico e Tecnológico (CNPq).

Declaração: os autores declaram não haver conflitos de interesse científico neste artigo.

\section{REFERÊNCIAS}

1. Kendall B, Esron R. Exercise-induced muscle damage and the potential protective role of estrogen. Sports Med. 2002;32(2):103-23.

2. Bulun SE, Adashi EY. The physiology and pathology of the female reproductive axis. In: Wilson JD, Foster DW, editors. Williams Textbook of Endocrinology. 11th ed. Philadelphia: WB Saunders Company; 2003. p. 541-614.

3. Richard D, Rochon L, Deshaies $Y$. Effects of exercise training on energy balance of ovariectomized rats. Am J Physiol. 1987;253(5 Pt 2):R740-5.

4. Kimura M, Irahara M, Yasui T, Saito S, Tezuka M, Yamano S, et al. The obesity in bilateral ovariectomized rats is related to a decrease in the expression of leptin receptors in the brain. Biochem Biophys Res Commun. 2002;290(4):1349-53.

5. Meli R, Pacilio M, Raso GM, Esposito E, Coppola A, Nasti A, et al. Estrogen and raloxifene modulate leptin and its receptor in hypothalamus and adipose tissue from ovariectomized rats. Endocrinology. 2004;145(7):3115-21.

6. Marassi MP, Fortunato RS, da Silva AC, Pereira VS, Carvalho DP, Rosenthal $D$, et al. Sexual dimorphism in thyroid function and type 1 iodothyronine deiodinase activity in pre-pubertal and adult rats. J Endocrinol. 2007;192(1):121-30.

7. Percegoni N, Ferreira AC, Rodrigues CF, Rosenthal D, Castelo Branco MT, Rumjanek VM, et al. Profile of serum IL-1 beta and IL-10 shortly after ovariectomy and estradiol replacement in rats. Horm Metab Res. 2009;41(1):50-4.

8. Heine PA, Taylor JA, Iwamoto GA, Lubahn DB, Cooke PS. Increased adipose tissue in male and female estrogen receptor $\alpha$ knockout mice. Proc Natl Acad Sci U S A. 2000;97(23):12729-34.
9. Jones ME, Thorburn AW, Britt KL, Hewitt KN, Wreford NG, Proietto $\mathrm{J}$, et al. Aromatase-deficient (ArKO) mice have a phenotype of increased adiposity. Proc Natl Acad Sci U S A. 2000:97(23):12735-40.

10. Richard D. Effects of ovarian hormones on energy balance and brown adipose tissue thermogenesis. Am J Physiol. 1986:250(2 Pt 2):R245-9.

11. Thompson EA Jr, Siiteri PK. The involvement of human placental microssomal cytochrome P-450 in aromatization. J Biol Chem. 1974:249(17):5373-8.

12. Simpson ER, Clyne C, Rubin G, Boon WC, Robertson K, Britt K, et al. Aromatase--a brief overview. Annu Rev Physiol. 2002:64:93-127.

13. Fisher CR, Graves KH, Parlow AF, Simpson ER. Characterization of mice deficient in aromatase (ArKO) because of targeted disruption of the cyp19 gene. Proc Natl Acad Sci U S A. 1998;95(12):6965-70.

14. Jones ME, McInnes KJ, Boon WC, Simpson ER. Estrogen and adiposity--utilizing models of aromatase deficiency to explore the relationship. J Steroid Biochem Mol Biol. 2007;106 (1-5):3-7.

15. Simpson ER, Misso M, Hewitt KN, Hill RA, BoonWC, Jones ME, et al. Estrogen--the good, the bad, and the unexpected. Endocr Rev. 2005;26(3):322-30.

16. Rochira V, Balestrieri A, Madeo B, Zirilli L, Granata A, Carani C. Osteoporosis and male age-related hypogonadism: role of sex steroids on bone (patho)physiology. Eur J Endocrinol. 2006;154(2):175-85.

17. Maffei L, Murata Y, Rochira V, Tubert G, Aranda C, Vasquez M, et al. Dysmetabolic syndrome in a man with a novel mutation of the aromatase gene: effects of testosterone, alendronate and estradiol treatment. J Clin Endocrinol Metab. 2004;89(1):61-70.

18. Jones ME, Boon WC, McInnes K, Maffei L, Carani C, Simpson ER. Recognizing rare disorders: aromatase deficiency. Nat Clin pract Endocrinol Metab. 2007;3(5):414-21.

19. Pitteloud N, Dwyer AA, DeCruz S, Lee H, Boepple PA, Crowley WF Jr, et al. Inhibition of luteinizing hormone secretion by testosterone in men requires aromatization for its pituitary but not its hypothalamic effects: evidence from the tandem study of normal and gonadotropin-releasing hormone-deficient men. J Clin Endocrinol Metab. 2008;93(3):784-91.

20. Rochira V, Balestrieri A, Madeo B, Spaggiari A, Carani C. Congenital estrogen deficiency in men: a new syndrome with different phenotypes; clinical and therapeutic implications in men. Mol Cell Endocrinol. 2002;193(1-2):19-28.

21. Lanfranco F, Zirilli L, Baldi M, Pignatti E, Corneli G, Ghigo E, et al. A novel mutation in the human aromatase gene: insights on the relationship among serum estradiol, longitudinal growth and bone mineral density in an adult man under estrogen replacement treatment. Bone. 2008;43(3):628-35.

22. Pardini D. Menopausal hormone therapy. Arq Bras Endocrinol Metabol. 2007;51(6):938-42.

23. Silva HG, Mendonça LM, Conceição FL, Zahar SE, Farias ML. Influence of obesity on bone density in postmenopausal women. Arq Bras Endocrinol Metab. 2007;51(6):943-9.

24. Mangelsdorf DJ, Thummel C, Beato M, Herrlich P, Schütz G, Umesono $\mathrm{K}$, et al. The nuclear receptor superfamily: the second decade. Cell. 1995;83(6):835-9.

25. Safe S, Kim K. Non-classical genomic estrogen receptor (ER)/specificity protein and ER/activating protein-1 signaling pathways. J Mol Endocrinol. 2008; 41(5):263-75.

26. Kuiper GG, Carlsson B, Grandien K, Enmark E, Häggblad J, Nilsson $\mathrm{S}$, et al. Comparison of the ligand binding specificity and transcript tissue distribution of estrogen receptors alpha and beta. Endocrinology. 1997;138(3):863-70.

27. Carr MC. The emergence of the metabolic syndrome with menopause. J Clin Endocrinol Metab. 2003;88(6):2404-11.

28. Musatov S, Chen W, Pfaff DW, Mobbs CV, Yang XJ, Clegg DJ, et al. Silencing of estrogen receptor $\alpha$ in the ventromedial nucleus of 
hypothalamus leads to metabolic syndrome. Proc Natl Acad Sci U S A. 2007;104(7):2501-6.

29. Ohlsson C, Hellberg N, Parini P, Vidal O, Bohlooly-Y M, Rudling $M$, et al. Obesity and disturbed lipoprotein profile in estrogen receptor-alpha-deficient male mice. Biochem Biophys Res Commun. 2000; 278(3):640-5.

30. Foryst-Ludwig A, Clemenz M, Hohmann S, Hartge M, Sprang C, Frost N, et al. Metabolic actions of estrogen receptor beta (ERbeta) are mediated by a negative cross-talk with PPARgamma. PLoS Genet. 2008;4(6):e1000108.

31. Barros RP, Machado UF, Gustafsson JA. Estrogen receptors: new players in diabetes mellitus. Trends Mol Med. 2006;12(9):425-31.

32. Naaz A, Zakroczymski M, Heine P, Taylor J, Saunders $P$, Lubahn $D$, et al. Effect of ovariectomy on adipose tissue of mice in the absence of estrogen receptor alpha $(E R \alpha)$ : a potential role for estrogen receptor beta (ER $\beta$ ). Horm Metab Res. 2002;34(11-12):758-63.

33. Villena JA, Hock MB, Chang WI, Barcas JE, Giguère $V$, Kralli $A$. Orphan nuclear receptor estrogen-related receptor $\alpha$ is essential for adaptive thermogenesis. Proc Natl Acad Sci U S A. 2007;104(4):1418-23.

34. Mayes JS, Watson GH. Direct effects of sex steroid hormones on adipose tissues and obesity. Obes Rev. 2004;5(4):197-216.

35. Hong SC, Yoo SW, Cho GJ, Kim T, Hur JY, Park YK, Lee KW, Kim $\mathrm{SH}$. Correlation between estrogens and serum adipocytokines in premenopausal and postmenopausal women. Menopause. 2007;14(5):835-40.

36. Matsubara M, Maruoka S, Katayose S. Inverse relationship between plasma adiponectin and leptin concentrations in normalweight and obese women. Eur J Endocrinol. 2002;147(2):173-80.

37. Steppan CM, Bailey ST, Bhat S, Brown EJ, Banerjee RR, Wright $\mathrm{CM}$, et al. The hormone resistin links obesity to diabetes. Nature. 2001; 409(6818):307-12.

38. Vendrell J, Broch M, Vilarrasa N, Molina A, Gómez JM, Gutiérrez $C$, et al. Resistin, adiponectin, ghrelin, leptin, and proinflammatory cytokines: relationships in obesity. Obes Res. 2004;12(6):962-71.

39. Kohrt WM, Ehsani AA, Birge SJ Jr. HRT preserves increases in bone mineral density and reductions in body fat after a supervised exercise program. J Appl Physiol. 1998;84(5):1506-12.

40. Rebuffé-Scrive M, Eldh J, Hafström LO, Björntorp P. Metabolism of mammary, abdominal, and femoral adipocytes in women before and after menopause. Metabolism. 1986;35(9):792-7.

41. James W. Epidemiology of obesity. Int J Obes Relat Metab Disord. 1992; Suppl 2:S23-6.

42. Levine JA, Eberhardt NL, Jensen MD. Role of nonexercise activity thermogenesis in resistance to fat gain in humans. Science. 1999;283(5399):212-4.

43. Tou CL, Wade CE. Determinants affecting physical activity levels in animal models. Exp Biol Med. 2002;227(8):587-600.

44. Jéquier E, Felber JP. Indirect calorimetry. Baillieres Clin Endocrinol Metab. 1987;1(4):911-35.
45. Balady GJ, Berra KA, Golding LA. Diretrizes do ACSM para osTestes de Esforço e sua Prescrição. $6^{a}$ ed. Rio de Janeiro: Guanabara Koogan SA; 2003.

46. Ardevol A, Adan C, Remesar X, Fernández-López JA, Alemany M. Hind leg heat balance in obese Zucker rats during exercise. Pflugers Arch. 1998;435(4):454-64.

47. Poehlman ET, Toth MJ, Gardner AW. Changes in energy balance and body composition at menopause: a controlled longitudinal study. Ann Intern Med. 1995;123 (9):673-5.

48. McTiernan A, Stanfors JL, Daling JR. Prevalence and correlates of recreational physical activity in women aged $50-64$ years. Menopause. 1998;5(2):95-101.

49. Calles-Escandon J, Arciero PJ, Gardner AW, Bautman C, Poehlman ET. Basal fat oxidation decreases with aging in women. $J$ Appl Physiol. 1995;78(1):266-71.

50. Korth WM, Ehsani AA, Birge SJ Jr. HRT preserves increases in bone mineral density and reductions in body fat after a supervised exercise program. J Appl Physiol. 1998; 84(5):1506-12.

51. Pedersen SB, Bruun JM, Kristensen K, Richelsen B. Regulation of UCP1, UCP2, and UCP3 m RNA expression in brown adipose tissue, white adipose tissue, and skeletal muscle in rats by estrogen. Biochem Biophys Res Commun. 2001;288(1):191-7.

52. Tropp J, Markus E. Sex differences in the dynamics of cue utilization and exploratory behavior. Behav Brain Res. 2001;119(2):14354.

53. Gentry RT, Wade GN. Sex differences in sensitivity of food intake, body weight, and running-wheel activity to ovarian steroids in rats. J Comp Physiol Psychol. 1976;90(8):747-54.

54. ShimomuraY, Shimizu H,Takashashi M, Sato N, UeharaY, Fukatsu $A$, et al. The significance of decreased ambulatory activity during the generation by long-term observation of obesity in ovariectomized rats. Physiol Behav. 1990;47(1):155-9.

55. Novak CM, Kotz CM, Levine JA. Central orexin sensitivity, physical activity, and obesity in diet-induced obese and diet-resistant rats. Am J Physiol Endocrinol Metab. 2006;290(2):E 396-403.

56. Novak CM, Zhang CM, Levine JA. Sensitivity of the hypothalamic paraventricular nucleus to the locomotor- activating effects of neuromedin U in obesity. Brain Res. 2007:1169; 57-68.

57. Latour MG, Shinoda M, Lavoie JM. Metabolic effects of physical training in ovariectomized and hyperestrogenic rats. J Appl Physiol. 2001;90(1):235-41.

58. Melton SA, Hegsted M, Keenan MJ, Zhang Y, Morris S, Potter Bulot $\mathrm{L}$, et al. Swimming eliminates the weight gain and abdominal fat associated with ovariectomy in the retired breeder rat despite high-fat diet selection. Appetite. 2000;35(1):1-7.

59. Poehlman ET. Menopause, energy expenditure and body composition. Acta Obstet Gynecol Scand. 2002;81(7):603-11.

60. Levine JA, Lanningham-Foster LM, McCrady SK, Krizan AC, Olson $\mathrm{LR}$, Kane $\mathrm{PH}$, et al. Interindividual variation in posture allocation: possible role in human obesity. Science. 2005;307(5709):584-6. 